\title{
Non-Markovian Quantum Stochastic Processes and Their Entropy
}

\author{
Göran Lindblad \\ Department of Theoretical Physics, Royal Institute of Technology, \\ S-100 44 Stockholm 70, Sweden
}

\begin{abstract}
A definition of a quantum stochastic process (QSP) in discrete time capable of describing non-Markovian effects is introduced. The formalism is based directly on the physically relevant correlation functions. The notion of complete positivity is used as the main mathematical tool. Two different but equivalent canonical representations of a QSP in terms of completely positive maps are derived. A quantum generalization of the Kolmogorov-Sinai entropy is proved to exist.
\end{abstract}

\section{Introduction}

The purpose of this paper is to introduce the notion of a quantum stochastic process (QSP) and to define the entropy of a stationary QSP with a normal invariant state. Our formalism is intended to describe the irreversible time evolution of a finite open quantum system in contact with arbitrary reservoirs and measuring instruments. This time evolution is not assumed to be Markovian, i.e. the system has a memory.

Our definition of a QSP differs from that of E. B. Davies, which includes a Markov condition [1]. In fact most previous work on the dynamics of open quantum systems have treated the Markovian case only. Examples of recent work on Markovian systems are Davies' rigorous results on Markovian master equations [2], the theory of dynamical semigroups [3], and the derivation of the general form of their generators [4-6].

The abstract projection method introduced by Nakajima and Zwanzig, however, leads to generalized master equations (GME) which are non-Markovian in general [7-9]. The exact solution of the GME is equivalent to the complete description of the dynamics of the total system including reservoirs. Approximate treatments suffer from the defect that conditions sufficient to ensure the positivity preserving property of the time evolution are not known. Consequently it is difficult to stop negative probabilities from cropping up [10]. The alternative approach of generalizing the Langevin method to the non-commutative case also runs into severe difficulties [11].

In the commutative case the theory of stochastic processes gives a sound basis for discussions of similar fundamental problems. Therefore it seems worthwhile to 
attempt the design of a formalism for QSPs of the generality needed to include arbitrary memory effects.

In the theory of Markovian QSPs referred to above the stochastic element is given by dynamical maps on the set of states (or on the observables) of the system. These maps are the generalizations of stochastic matrices in the commutative case. When attempting an extension to non-Markovian processes it is not obvious which objects one should generalize. It is well known that in the commutative case the definition of a random process with a discrete time parameter by joint probabilities is equivalent to giving a state on a classical system with configuration space $\mathbb{Z}$. This structure is easily generalized to the concept of a random field on any configuration space (see e.g. [12]). In the quantum case the time and configuration space parameters are not equivalent as joint states for two times cannot be defined even in the Markovian case [13]. Accardi tried to avoid this difficulty by modifying the notions of a Markov system and conditional expectation [14]. We do not want to take this way out because the theory of Markovian systems referred to above is satisfactory and should be retained as a special case of the general theory.

We choose to generalize the concept of a dynamical map to take into account the memory effects. In $\S 2$ a QSP is defined as a family of dynamical maps connecting the observables relating to several instants of time (which we will choose to be discrete in this paper). These maps are closely related to the correlation functions which are physically relevant e.g. for the theory of coherence in quantum optics. In this definition we have to introduce at every instant a set of operations resulting from the instantaneous interaction of the system with an arbitrary external apparatus. We assume that the dynamical maps and the operations are given by normal completely positive (CP) maps on the algebra of observables [which will be $B(\mathscr{H})$ in this paper].

In $\S 3$ a representation of the family of CP maps defining a QSP is described. A consequence of this result is that a QSP can be derived from the interaction of the system with a reservoir. The result of $\S 4$ describes a QSP as a CP map on an infinite tensor product of algebras and clarifies the convex structure of the set of QSPs. This construction allows us to define the entropy of a stationary QSP with a normal invariant state in $\S 5$.

The problem of defining a QSP and its entropy when the algebra of observables is a subalgebra of $B(\mathscr{H})$ or when the time parameter is continuous will be left for future publications, as well as a detailed comparison of our formalism with the commutative case. There is a special class of QSPs which can be given a complete description, namely QSPs (in continuous time) defined by Gaussian (quasifree) CP maps on the CCR algebra. An account of this work will be published elsewhere.

\section{Definition of a QSP}

We consider a quantum system $\mathrm{S}$ whose algebra of observables is $\mathscr{A}=B(\mathscr{H}), \mathscr{H}$ a separable Hilbert space. The set of normal states on $B(\mathscr{H})$ (the set of density operators $\varrho \geqq 0 \operatorname{Tr} \varrho=1)$ is denoted by $S(\mathscr{H})$. The time parameter is taken to be discrete. For each $t=n \in \mathbb{Z}$ we have an algebra $\mathscr{A}(n) \simeq \mathscr{A}$ and an arbitrary instrument $\mathscr{E}_{n}$ in the following class. 
Definition 2.1. Let $\Omega$ be a countable set (the set of outcomes). An instrument on the system $S$ is a map $\mathscr{E}$ from subsets of $\Omega$ to $\mathrm{CP}(\mathscr{A})_{\sigma}$

$$
\begin{aligned}
& \mathscr{E}(E)[X]=\sum_{\omega \in E} V(\omega)^{+} X V(\omega), \\
& \mathscr{E}(\Omega)[I]=I, \quad E \subset \Omega .
\end{aligned}
$$

The probabilistic interpretation is the following: If the initial state of $S$ is $\varrho$ then the probability of observing that the outcome is in $E \subset \Omega$ when reading the instrument is $p(E)=\varrho(\mathscr{E}(E)[I])$ and the state after this observation is $p(E)^{-1} \mathscr{E} *(E)[\varrho]$.

Compared with the definition in [1] we have restricted ourselves to countable $\Omega$ and CP maps. See [4] for the reasons for choosing CP maps and the notations used here and the Appendix for some properties of CP maps. * denotes the dual map on $S(\mathscr{H})$.

By definition a Markovian dynamics of the system is given by a family of maps $T_{n}: \mathscr{A}(n) \rightarrow \mathscr{A}(n-1), T_{n} \in \mathrm{CP}(\mathscr{A})_{\sigma}$. Note that we "go backwards in time" in the Heisenberg picture. If the state of $S$ at $t=m$ is $\varrho$ then the probability of obtaining a sequence $\left\{\omega_{k}: k=m+1, \ldots, n\right\}$ of instrument readings is

$$
\begin{aligned}
p\left(\left\{\omega_{k}\right\}\right)= & \varrho\left(T _ { m + 1 } \left[V ( \omega _ { m + 1 } ) ^ { + } T _ { m + 2 } \left[V\left(\omega_{m+2}\right)^{+}\right.\right.\right. \\
& \left.\left.\ldots T_{n-1}\left[V\left(\omega_{n-1}\right)^{+} T_{n}\left[V\left(\omega_{n}\right)^{+} V\left(\omega_{n}\right)\right] V\left(\omega_{n-1}\right)\right] \ldots V\left(\omega_{m+1}\right)\right]\right) .
\end{aligned}
$$

Note that the instruments $\mathscr{E}_{k}$ may be different but we skip the extra indices. Similar expressions for Markovian systems have been considered e.g. in [15], [16] Eq. 7.28, [9] Eq. 2e.17.

For a general QSP we retain the essentials of this structure by postulating for every sequence $\{t=m, m+1, \ldots, n\}$ a set of bounded normal CP maps of $\mathscr{A}(n)$ into $\mathscr{A}(m)$

$$
X \mapsto T_{(m, n)}\left[Y_{m+1}^{+}, \ldots, Y_{n-1}^{+} ; X ; Y_{n-1}, \ldots, Y_{m+1}\right]
$$

which depend sesquilinearly and norm continuously on the $Y_{k}$. The probabilistic interpretation is as follows: If the system is initially $(t=m)$ in the state $\varrho$ then the probability of obtaining a sequence of outcomes $\left\{\omega_{k}: k=m+1, \ldots, n\right\}$ when reading the instruments $\mathscr{E}_{k}$ is (by definition)

$$
p\left(\left\{\omega_{k}\right\}\right)=\varrho\left(T_{(m, n)}\left[V\left(\omega_{m+1}\right)^{+}, \ldots ; V\left(\omega_{n}\right)^{+} V\left(\omega_{n}\right) ; \ldots, V\left(\omega_{m+1}\right)\right]\right) .
$$

By polarization we obtain multilinear maps

$$
\begin{aligned}
& T_{(m, n)}: \stackrel{2(n-m)}{X} \mathscr{A} \rightarrow \mathscr{A}^{T_{(m, n)}}\left[\left\{X_{k}^{\prime} ; X_{k}\right\}_{m+1}^{n}\right] \equiv T_{(m, n)}\left[X_{m+1}^{\prime}, \ldots, X_{n-1}^{\prime} ; X_{n}^{\prime} X_{n} ; X_{n-1}, \ldots, X_{m+1}\right] .
\end{aligned}
$$

The $T_{(m, n)}\left[\left\{X_{k}^{\prime} ; X_{k}\right\}\right]$ we call correlation operators. The $T_{(m, n)}$ can be extended to multilinear maps on $M_{N}(\mathscr{A})=\mathscr{A} \otimes M_{N}(\mathbb{C})$ in an obvious way through

$$
\begin{aligned}
& T_{(m, n)}^{(N)}\left[\left\{X_{k}^{\prime} \otimes Y_{k}^{\prime} ; X_{k} \otimes Y_{k}\right\}\right]=T_{(m, n)}^{(N)}\left[\left\{X_{k}^{\prime} ; X_{k}\right\}\right] \otimes Y_{m+1}^{\prime} \ldots Y_{n}^{\prime} Y_{n} \ldots Y_{m+1} \\
& X_{k}^{\prime}, X_{k} \in \mathscr{A}, Y_{k}^{\prime}, Y_{k} \in M_{N}(\mathbb{C})
\end{aligned}
$$


plus linearity. As shown in [4] $\S 2$ the maps $T^{(N)}$ represent (up to unitary isomorphism) the dynamics of a noninteracting system composed of $S$ and a system $S^{\prime}$ with reversible (Hamiltonian) dynamics. Consistency demands that the $T^{(N)}$ are positive for all $N$. This condition is the complete positivity of the $T_{(m, n)}$ which can be written

$$
\sum_{\alpha . \beta} X_{m}(\alpha)^{+} T_{(m . n)}\left[\left\{X_{k}(\alpha)^{+} ; X_{k}(\beta)\right\}_{m+1}^{n}\right] X_{m}(\beta) \geqq 0
$$

for all $\left\{X_{k}(\alpha): k=m, \ldots, n, \alpha=1, \ldots, N\right\}$ and all $N$.

The maps $T_{(m, n)}$ are not independent. When we sum over all outcomes of the reading of the last instrument in the sequence $\left\{\mathscr{E}_{k}\right\}_{m+1}^{n}$ the result is the same as reading the instruments $\left\{\mathscr{E}_{k}\right\}_{m+1}^{n-1}$ only:

$$
\sum_{\omega_{n} \in \Omega} p\left(\omega_{m+1}, \ldots, \omega_{n-1}, \omega_{n}\right)=p\left(\omega_{m+1}, \ldots, \omega_{n-1}\right) .
$$

Note that the summing over $\omega_{k}, k<n$, does not give a similar relation. Performing a measurement without reading the result does influence the results of later measurements but not those of earlier ones.

The validity of (2.3) for any choice of $\mathscr{E}_{n}$ shows that $T_{(m, n)}$ can indeed only depend on $X_{n}^{\prime}, X_{n}$ through the combination $X_{n}^{\prime} X_{n}$ as we have already assumed. From (2.3) we find that the following compatibility relation must hold for all $m<n$

$$
\begin{aligned}
& T_{(m, n)}\left[\left\{X_{k}^{+} ; X_{k}\right\}_{m+1}^{n}: X_{n}=I\right]=T_{(m, n-1)}\left[\left\{X_{k}^{+} ; X_{k}\right\}_{m+1}^{n-1}\right] \\
& T_{(m, m+1)}[I]=I .
\end{aligned}
$$

The set $\left\{T_{(m, n)}\right\}$ is called compatible if the conditions (2.4) are satisfied. We will see below that the compatibility conditions and the normality in $X_{n}^{+} X_{n}$ of $T_{(m, n)}$ means that the $T_{(m, n)}$ are ultrastrongly continuous in all $X_{k}$. We express this by saying that the $T_{(m, n)}$ are normal.

Definition 2.2. (a) A QSP on $\mathscr{A}$ is a compatible set of normal CP maps $T_{(m, n)}:{ }^{2(m-n)} X^{-A} \rightarrow \mathscr{A}, m<n$, i.e. satisfying (2.2), (2.4).

(b) The state $\varrho$ is called invariant if for all $\left\{X_{k}^{\prime}, X_{k}\right\}, m<n$,

$$
\varrho\left(T_{(m, n)}\left[\left\{X_{k}^{\prime} ; X_{k}\right\}_{m+1}^{n}\right]\right)=\varrho\left(X_{m+1}^{\prime} T_{(m+1, n)}\left[\left\{X_{k}^{\prime} ; X_{k}\right\}_{m+2}^{n}\right] X_{m+1}\right) .
$$

(c) The QSP is said to be stationary if $T_{(m, n)}=T_{(0, n-m)}$ for all $m<n$ and if it has a faithful normal invariant state.

Example. Let $\mu_{(m, n)}, m<n$, be probability measures on $\mathbb{R}^{n-m+1}$ defining a stochastic process on $\mathbb{R}$ (with discrete time parameter) and let $U(s), s \in \mathbb{R}$, be a strongly continuous group of unitary operators in $\mathscr{H}$. Then we can define a QSP by

$$
\begin{aligned}
& T_{(m, n)}\left[\left\{X_{k}^{\prime} ; X_{k}\right\}_{m+1}^{n}\right]=\int d \mu_{(m, n)}\left(s_{m}, \ldots, s_{n}\right) U\left(s_{m+1}-s_{m}\right)^{+} X_{m+1}^{\prime} U\left(s_{m+2}-s_{m+1}\right)^{+} \\
& \ldots X_{n-1}^{\prime} U\left(s_{n}-s_{n-1}\right)^{+} X_{n}^{\prime} X_{n} U\left(s_{n}-s_{n-1}\right) X_{n-1} \\
& \ldots U\left(s_{m+2}-s_{m+1}\right) X_{m+1} U\left(s_{m+1}-s_{m}\right) .
\end{aligned}
$$

$\varrho$ is an invariant state for the process if it is invariant under $U(s)$. The process is stationary if the commutative SP defined by the $\mu_{(m, n)}$ is stationary. 
Remarks. 1. The definition of a QSP with a continuous time parameter needs an additional compatibility condition : for all sequences $\left\{t_{0} \leqq t_{1} \leqq \ldots \leqq t_{n}\right\},\left\{X\left(t_{k}\right)\right\}_{1}^{n}$ with $X\left(t_{m}\right)=I$ for some $m<n$

$$
T\left\{t_{k}\right\}\left[\left\{X\left(t_{k}\right)^{+} ; X\left(t_{k}\right)\right\}: X\left(t_{m}\right)=I\right]=T\left\{t_{k}: k \neq m\right\}\left[\left\{X\left(t_{k}\right)^{+} ; X\left(t_{k}\right)\right\}: k \neq m\right] .
$$

2. The name correlation operators has been chosen as their expectation values correspond to commutative correlation functions. E.g. : the correlation function $E\left\{X_{0}\left(t_{0}\right) X_{1}\left(t_{1}\right) X_{2}\left(t_{2}\right)\right\}\left(t_{0} \leqq t_{1} \leqq t_{2}\right)$ has the non-commutative counterpart

$$
\varrho\left(X_{0} T\left\{t_{0}, t_{1}, t_{2}\right\}\left[X_{1} ; X_{2} ; I\right]\right) \text {. }
$$

We can similarly find any non-commutative correlation function with a time ordering of the type $t_{0} \leqq t_{1} \leqq \ldots \leqq t_{k} \geqq t_{k+1} \geqq \ldots \geqq t_{n}$ (for some $k$ ). If the process is stationary with invariant state $\varrho$ then it follows from (2.2) that the function

$$
R(t ; X, Y)=\varrho(X T\{0, t\}[Y]) \quad(t \geqq 0), \quad=\varrho(T\{0,-t\}[X] Y) \quad(t<0)
$$

is positive definite in the sense that

$$
\sum_{\alpha, \beta} \lambda_{\alpha}^{*} \lambda_{\beta} R\left(t_{\alpha}-t_{\beta} ; X_{\alpha}^{+}, X_{\beta}\right) \geqq 0
$$

for every sequence $\left\{t_{\alpha} \in \mathbb{R}, \lambda_{\alpha} \in \mathbb{C}, X_{\alpha} \in \mathscr{A}\right\}_{1}^{N}$ and all $N$.

3. Multitime correlation functions for quantum systems with non-Markovian dynamics has been considered e.g. in $[9,17,18]$. They were derived from a GME and thus inherit the problems indicated in $\S 1$. The approach used by Bausch and Stahl in [19] is similar in spirit to ours as it only refers to quantities belonging to the small system $S$ and imposes positivity properties. But as they did not use the property of complete positivity they could not obtain the structure described below.

\section{Canonical Representation of a QSP}

We want to give a representation of a QSP which is a generalization of the Stinespring representation of a CP map. We will consider only stationary QSPs in the following, hence it is enough to define the maps $\left\{T_{(0, n)}\right\}$.

Theorem 3.1. (a) If $\left\{T_{(0, n)}\right\}$ define a QSP then there are normal $*$-representations $\pi_{n}$ of $\mathscr{A}$ in Hilbert spaces $\mathscr{K}_{n}$ and isometric maps $V_{n} \in B\left(\mathscr{K}_{n-1}, \mathscr{K}_{n}\right)\left(\mathscr{K}_{0}=\mathscr{H}\right)$ such that for all $n$

$$
\begin{aligned}
& T_{(0, n)}\left[\left\{X_{k}^{+} ; X_{k}\right\}_{1}^{n}\right]=W_{(0, n)}\left[\left\{X_{k}\right\}_{1}^{n}\right]^{+} W_{(0, n)}\left[\left\{X_{k}\right\}_{1}^{n}\right] \\
& W_{(0, n)}\left[\left\{X_{k}\right\}_{1}^{n}\right]=\pi_{n}\left(X_{n}\right) V_{n} \pi_{n-1}\left(X_{n-1}\right) \ldots \pi_{1}\left(X_{1}\right) V_{1} .
\end{aligned}
$$

The representation is unique up to unitary equivalence if

$$
\mathscr{K}_{n}=\left[W_{(0, n)}\left[\mathcal{X}^{n} \mathscr{A}\right] \mathscr{H}\right] .
$$

Conversely, each set $\left\{T_{(0, n)}\right\}$ of the form (3.1) satisfies all conditions for a stationary QSP save the existence of a normal invariant state. 
(b) Under the same conditions there is a normal $*$-representation $\pi$ of $\mathscr{A}$ in a Hilbert space $\mathscr{K}$, an isometry $V \in B(\mathscr{K})$ and an injection $p: \mathscr{H} \rightarrow \mathscr{K}$ such that

$$
T_{(0, n)}\left[\left\{X_{k}^{+} ; X_{k}\right\}_{1}^{n}\right]=p^{+} V^{+} \pi\left(X_{1}\right)^{+} V^{+} \ldots V^{+} \pi\left(X_{n}^{+} X_{n}\right) V \ldots V \pi\left(X_{1}\right) V p .
$$

Proof. (a) A repeated application of Lemma A.1 (a) using the substitution

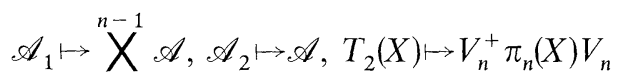

gives the statement. The converse is obvious.

(b) Put $\mathscr{K}=\oplus \mathscr{K}_{n}$ and define $\pi: \mathscr{A} \rightarrow B(\mathscr{K}), V \in B(\mathscr{K})$ and $p \in B(\mathscr{H}, \mathscr{K})$ through

$$
\begin{aligned}
& (\pi(X))_{i j}=\delta_{i j} \pi_{i}(X), \quad \pi_{0}=0, \\
& (V)_{i j}=\delta_{i+1, j} V_{j}, \quad(p)_{i}=\delta_{i 0} I .
\end{aligned}
$$

(3.3) says that the QSP has a Markovian dilation which is isometric and consequently there is also a unitary dilation. Compare the isometric dilations in the Markov case in [1] Theorem 9.4.3 and [20,21].

The results of this paragraph are valid for any $W^{*}$-algebra $\mathscr{A}$. The interpretation of $\left\{T_{(0, n)}\right\}$ as a QSP on $\mathscr{A}$, however, demands further conditions which we will not go into here.

When $\mathscr{A}=B(\mathscr{H})$ we can use (A.2) and write

$$
\pi_{n}(X)=X \otimes I \in \mathscr{A} \otimes B\left(h_{n}\right), V_{n} \in B\left(\mathscr{K}_{n-1}, \mathscr{K}_{n}\right)=\mathscr{A} \otimes B\left(h_{n-1}, h_{n}\right) .
$$

When (3.2) holds, then the remaining arbitrariness in the representation is given by (A.2) and (A.3)

$$
V_{n} \mapsto W_{n} V_{n} W_{n-1}^{+}, \quad W_{n} \text { unitary } \in I \otimes B\left(\hbar_{n}\right) .
$$

\section{Tensor Product Representation}

We want to derive from a stationary QSP a set of normal CP maps $\left\{T_{n}\right\}$ on $W^{*}$-tensor products $\bigotimes^{n} \mathscr{A}$ where $\mathscr{A}=B(\mathscr{H})$ as before. We introduce the infinite tensor product [22]

$$
\mathscr{A}_{\infty}=\left[\bigcup_{v} \otimes \mathscr{A}\right],
$$

the natural injection

$$
\alpha: \otimes^{\nu} \mathscr{A} \rightarrow \mathscr{A}_{\nu} \subset \mathscr{A}_{\infty}, \quad \mathscr{A}_{0}=\mathbb{C} I
$$

satisfying $\alpha(X \otimes I)=\alpha(X)$, and the amplification

$$
\left.\beta: \mathscr{A}_{v} \rightarrow \alpha(I \otimes(\otimes) \mathscr{A})\right) \subset \mathscr{A}_{v+1}
$$

defined by $\beta(\alpha(X))=\alpha(I \otimes X)$. 
$\alpha$ and $\beta$ are $*$-isomorphisms, hence normal (for fixed $v$ ) CP maps and $[\alpha(X), \beta(Y)]=0$, all $X \in \mathscr{A}, Y \in \mathscr{A}_{\infty}$.

If the $*$-representations $\pi_{n}$ of $\mathscr{A}$ in (3.1) are chosen as in (3.4), then they can be extended to representations of $\mathscr{A}_{v}$

$$
\pi_{n}: \mathscr{A}_{v} \rightarrow \mathscr{A}_{v} \otimes B\left(h_{n}\right), \pi_{n}(X)=X \otimes I, X \in \mathscr{A}_{v},
$$

any $v$.

$\alpha$ and $\beta$ are also extended in the natural way for any $v, n$, e.g.

$\alpha:\left(\otimes^{v} \mathscr{A}\right) \otimes B\left(h_{n}\right) \rightarrow \mathscr{A}_{v} \otimes B\left(h_{n}\right)$

and similarly when $B\left(h_{n}\right)$ is replaced by $B\left(h_{n-1}, h_{n}\right)$. The use of the same notation for the extension should not cause any confusion. We find that

$$
\begin{aligned}
& \pi_{n}(\alpha(X))=\alpha\left(\pi_{n}(X)\right) \\
& \pi_{n}(\alpha(X)) \beta\left(\alpha\left(V_{n}\right)\right)=\beta\left(\alpha\left(V_{n}\right)\right) \pi_{n-1}(\alpha(X))
\end{aligned}
$$

for all $X \in \mathscr{A}$.

Given a QSP we can extend the maps $T_{(0, n)}$ to arguments in $\mathscr{A}_{\nu}$ of the form $X \beta(Y)$ $\left(X \in \mathscr{A}_{1}, Y \in \mathscr{A}_{v-1}\right)$ such that they operate only on $\mathscr{A}_{1}$ [cf. (2.1)]

$$
\begin{aligned}
& T_{(0, n)}\left[\left\{\alpha\left(X_{k}\right)^{+} \beta\left(Y_{k}\right)^{+} ; \alpha\left(X_{k}\right) \beta\left(Y_{k}\right)\right\}_{1}^{n}\right] \\
& =\alpha\left(T_{(0, n)}\left[\left\{X_{k}^{+} ; X_{k}\right\}_{1}^{n}\right]\right) \beta\left(Y_{1}^{+} \ldots Y_{n}^{+} Y_{n} \ldots Y_{1}\right)
\end{aligned}
$$

for all $\left\{X_{k} \in \mathscr{A}, Y_{k} \in \mathscr{A}_{v-1}\right\}$.

Using (3.1) we obtain the canonical form: for all $\left\{X_{k}\right\}$ of the product form

$$
T_{(0, n)}\left[\left\{X_{k}^{+} ; X_{k}\right\}_{1}^{n}\right]=\left[\prod_{k} \alpha\left(V_{k}\right)^{+} \pi_{k}\left(X_{k}\right)^{+}\right]\left[\prod_{l} \pi_{l}\left(X_{l}\right) \alpha\left(V_{l}\right)\right] .
$$

But the representation is then continued (through the $\pi_{n}$ ) in a unique way to $\left\{X_{k} \in \mathscr{A}_{v}\right\}$ for any $v$.

Consider the action of $\beta$ for given $v$. We choose a normal $\mathrm{CP}$ extension [the arbitrariness is given by Lemma A.1 (b)]

$$
\beta_{v}: \mathscr{A}_{v+1} \rightarrow \mathscr{A}_{v+1}
$$

such that $\beta_{v}\left|\mathscr{A}_{v}=\beta\right| \mathscr{A}_{v} . \beta_{v}$ is of the form (A.1)

$$
\beta_{v}(X)=\sum_{i} W_{v}(i)^{+} X W_{v}(i)
$$

where $X, W_{v}(i) \in \mathscr{A}_{v+1}$. The arbitrariness in the choice of $W_{v}$ is given by (A.3).

We will now show that the quantity

$$
\begin{aligned}
& T_{n}\left[\alpha\left(\bigotimes^{n} X_{k}^{+} X_{k}\right)\right] \\
& =\sum_{\left(i_{k}\right)} T_{(0, n)}\left[\left\{\alpha\left(X_{k}\right)^{+} W_{n-k}\left(i_{k}\right)^{+}\right\}_{1}^{n-1} ; \alpha\left(X_{n}^{+} X_{n}\right) ;\left\{W_{n-k}\left(i_{k}\right) \alpha\left(X_{k}\right)\right\}_{1}^{n-1}\right] \\
& \in \mathscr{A}_{n}, X_{k} \in \mathscr{A}
\end{aligned}
$$


is a function only of $\left\{X_{k}^{+} X_{k}\right\}_{1}^{n}$ as indicated. First we note, using (4.3), that it is independent of the arbitrariness in the choice of the $\beta_{v}$ [by Lemma A.1 (b)] and the $W_{v}$ [by (A.3)]. Furthermore, if $U \in \mathscr{A}_{1}$ is unitary then

$$
U^{+} \beta_{v}(X) U=\beta_{v}(X), \text { all } X \in \mathscr{A}_{v+1}
$$

and consequently $W_{v} U$ and $W_{v}$ are related by (A.3). From this and (4.3) follows that if $U_{k} \in \mathscr{A}$ are unitary, then (4.4) is invariant under the substitution $X_{k} \mapsto U_{k} X_{k}$. From the fact that every element in $\mathscr{A}$ is a finite linear combination of unitaries follows that (4.4) is a function of $\left\{X_{k}^{+} X_{k}\right\}_{1}^{n}$ only and consequently $T_{n}$ is well-defined on elements in $\mathscr{A}_{n}$ of the form $X=\bigotimes X_{k}$. If we use the representation (4.3) we find after some computation using (4.1) that

$$
\begin{aligned}
& T_{n}(X)=W_{n}^{+} \pi_{n}(X) W_{n} \\
& W_{n}=\beta^{n-1}\left[\alpha\left(V_{n}\right)\right] \beta^{n-2}\left[\alpha\left(V_{n-1}\right)\right] \ldots \beta\left[\alpha\left(V_{2}\right)\right] \alpha\left(V_{1}\right)
\end{aligned}
$$

for $X$ of product form. But $\pi_{n}$ and hence $T_{n}$ is continued in a unique way to $\mathscr{A}_{n}$ and, in the trivial way, to $\mathscr{A}_{v}, v>n$. $T_{n}$ is obviously a normal CP map on $\mathscr{A}_{n}$.

The compatibility relations have a very simple form for $\left\{T_{n}\right\}$. From (4.5) and the unitary of the $V_{k}$ follows that

$$
\begin{aligned}
& T_{n}(X)=T_{m}(X) \text { for } X \in \mathscr{A}_{m}, m<n \\
& T_{1}(I)=I .
\end{aligned}
$$

By norm continuity the set $\left\{T_{n}\right\}$ is extended in a unique way to a map $\operatorname{T} \in \operatorname{CP}\left(\mathscr{A}_{\infty}\right)$ which satisfies

$$
\mathbf{T}(X)=T_{n}(X) \text { for } X \in \mathscr{A}_{n} .
$$

From the construction (4.4) it is clear that the map $\left\{T_{(0, n)}\right\} \mapsto\left\{T_{n}\right\}$ defined on the convex set of QSPs is affine and preserves the partial order induced by the CP conditions. Conversely, given a set $\left\{T_{n} \in \mathrm{CP}\left(\mathscr{A}_{n}\right)_{\sigma}\right\}$ satisfying (4.6), we find from Lemma A.1 (b) that it has the form (4.5), i.e. it is in the range of this affine map. Furthermore, we can use Lemma A.1 (c) repeatedly through the substitution

$$
\mathscr{A}_{1} \mapsto \mathscr{A}, \mathscr{A}_{2} \mapsto \mathscr{A}_{n-1}
$$

to define a QSP $\left\{T_{(0, n)}^{\prime}\right\}$. The map $\left\{T_{n}\right\} \mapsto\left\{T_{(0, n)}^{\prime}\right\}$ defined on the convex set of families of normal CP maps satisfying (4.6) is affine and order-preserving. It is easily checked that this map is the inverse of the first one.

If $\varrho$ is an invariant normal state for the QSP $\left\{T_{(0, n)}\right\}$ then it is clear from $(2.5)$ and (4.2) that the extension of the QSP to $\mathscr{A}_{v}$ satisfies

$$
\operatorname{Tr}_{1}\left[\alpha(\varrho) T_{(0, n)}\left[\left\{X_{k}^{+} ; X_{k}\right\}_{1}^{n}\right]\right]=\operatorname{Tr}_{1}\left[\alpha(\varrho) X_{1}^{+} T_{(0, n-1)}\left[\left\{X_{k}^{+} ; X_{k}\right\}_{2}^{n}\right] X_{1}\right]
$$

for all $\left\{X_{k} \in \mathscr{A}_{v}\right\}$. From (4.4) follows that

$$
\operatorname{Tr}_{1}\left[\alpha(\varrho) T_{n}(\alpha(X) \beta(Y))\right]=\operatorname{Tr}_{1}\left[\alpha(\bar{\varrho}) \alpha(X) \beta\left(T_{n-1}(Y)\right)\right]=\bar{\varrho}(X) T_{n-1}(Y)
$$

for all $X \in \mathscr{A}, Y \in \mathscr{A}_{n-1}$. In terms of the dual maps $T_{n}^{*}: S\left(\mathscr{A}_{n}\right) \rightarrow S\left(\mathscr{A}_{n}\right)$ the invariance condition reads

$$
T_{n}^{*}(\bar{\varrho} \otimes \varrho)=\bar{\varrho} \otimes T_{n-1}^{*}(\varrho)
$$


for all $\varrho \in S\left(\mathscr{A}_{n-1}\right)$. Consequently for all $\varrho \in S\left(\mathscr{A}_{n-k}\right), k<n$

$$
T_{n}^{*}\left(\stackrel{\aleph}{\bigotimes}^{\varrho} \otimes \varrho\right)=\bigotimes^{k} \varrho \otimes T_{n-k}^{*}(\varrho) \text {. }
$$

Conversely (4.7) implies (2.5). We sum up the results in

Theorem 4.1. A stationary $Q S P$ on $\mathscr{A}=B(\mathscr{H})$ is equivalently described by a map $\mathrm{T} \in \mathrm{CP}\left(\mathscr{A}_{\infty}\right)$ leaving each $\mathscr{A}_{n}$ invariant and where each $T_{n}=\mathbf{T} \mid \mathscr{A}_{n}$ is normal. The equivalence is given by (3.1) and (4.5) and defines an affine order isomorphism of convex sets with the partial order defined by the CP conditions. $\varrho$ is an invariant state iff (4.7) holds.

Remarks. 1. We note that the QSP is Markovian iff $T_{n}=\bigotimes^{n} T_{1}$. Because of the tensor product form it is easy to see that it is extreme (ergodic) in the convex set of QSPs. Consequently there is a considerable difference in structure between commutative and non-commutative Markov processes. In fact the Markov QSPs are in some respects more similar to the subclass of Bernoulli SPs.

2. It follows easily from Lemma A.2 that there is still another description of a QSP as a quasilocal quantum lattice system with each lattice point having the Hilbert space $\mathscr{H} \otimes \mathscr{H}$. The conditions (4.6) and (4.7) are not more perspicuous in this formulation, however, and we will not write them down. We will use this fact only to show the existence of a mean entropy in $\S 5$.

\section{The Entropy of a Stationary QSP}

We first define the entropy of a map $T \in \mathrm{CP}(\mathscr{H})_{\sigma}, T(I)=I$ with an invariant normal state $\varrho: T^{*}(\varrho)=\varrho \in S(\mathscr{H})$. We can realize $T$ through an isometric dilation on a larger system $S+R$ where $R$ is a reservoir described by a Hilbert space $\mathscr{H}_{R}$ as described in [4].

If for any state $\varrho_{R}$ of $R$ we take $\varrho \otimes \varrho_{R}$ to be the initial state of $S+R$ then the final state of $S$ is $\varrho$ and that of $R$ is (with the notation of (A.1) and [4] Proposition 2)

$$
\varrho_{R}^{\prime}=\sum_{i, j} \operatorname{Tr}\left[V_{i} \varrho V_{j}^{+}\right] W_{i} \varrho_{R} W_{j}^{+} .
$$

If the entropy is defined as usual $(S(\varrho)=-\operatorname{Tr}[\varrho \ln \varrho])$ then

$$
\begin{aligned}
& S\left(\varrho_{R}^{\prime}\right)-S\left(\varrho_{R}\right)=S[\sigma(T, \varrho)] \\
& \sigma(T, \varrho)_{i j}=\operatorname{Tr}\left[V_{i} \varrho V_{j}^{+}\right] .
\end{aligned}
$$

This is shown by introducing a Hilbert space $h$ with a complete orthonormal set $\{|i\rangle\}$ and defining an isometric map $W: \mathscr{H}_{R} \rightarrow \mathscr{H}_{R} \otimes h$ through

$$
W|\phi\rangle=\sum_{i} W_{i}^{+}|\phi\rangle \otimes|i\rangle, \quad \text { all }|\phi\rangle \in \mathscr{H}_{R}
$$

Then (5.1) follows by the isometric invariance of entropy from

$$
W \varrho_{R}^{\prime} W^{+}=\varrho_{R} \otimes \sum_{i, j} \sigma(T, \varrho)_{i j}|i\rangle\langle j|
$$


$S[\sigma(T, \varrho)]$ depends only on $T$ and $\varrho$ and not on the representation $(\pi, V)$. In fact, according to (A.2), (A.3) equivalent representations give rise to unitary maps $\sigma(T, \varrho) \mapsto \sigma(T, \varrho)^{\prime}$ which conserve entropy. Therefore we take the entropy of $T$ in the state $\varrho$ to be $S[\sigma(T, \varrho)]$ :

Definition 5.1. $S(T, \varrho)=S[\sigma(T, \varrho)]$.

There is an alternative description of the entropy which will prove convenient. From Lemma A. 2 we know that there is a pure state $\tau(\varrho) \in S\left(\mathscr{H}_{1} \otimes \mathscr{H}_{2}\right)$ where $\mathscr{H}_{1} \simeq \mathscr{H}_{2} \simeq \mathscr{H}$ such that $\operatorname{Tr}_{1} \tau(\varrho) \simeq \operatorname{Tr}_{2} \tau(\varrho) \simeq \varrho$. Consider the pure state

$$
\mu=(V \otimes I) \tau(\varrho)(V \otimes I)^{+} \in S\left(\mathscr{H}_{1} \otimes \mathscr{H}_{2} \otimes \mathscr{H}_{3}\right)
$$

where $\mathscr{H}_{3}=h$ in the notation of the Appendix. We find that if $\tau(T, \varrho)$ is defined as in Lemma A.2

$$
\begin{aligned}
& \operatorname{Tr}_{3} \mu=\left(T^{*} \otimes I\right) \tau(\varrho)=\tau(T, \varrho) \in S\left(\mathscr{H}_{1} \otimes \mathscr{H}_{2}\right) \\
& \operatorname{Tr}_{12} \mu=\sigma(T, \varrho) \in S\left(\mathscr{H}_{3}\right) .
\end{aligned}
$$

From the triangle inequality ([23] Eq. (2.13)) follows that $S[\tau(T, \varrho)]=S[\sigma(T, \varrho)]$ and we have proved

Theorem 5.2. $S(T, \varrho)=S[\tau(T, \varrho)]$.

The properties of $S(T, \varrho)$ follow from the affine correspondence of Lemma A.2 and the properties of $S(\tau)$ given e.g. in [23]. We only mention without proof the inequality $S(T, \varrho) \leqq 2 S(\varrho)$.

We now define the entropy of a stationary QSP with an invariant normal state $\varrho$ using the tensor product form of Theorem 4.1 .

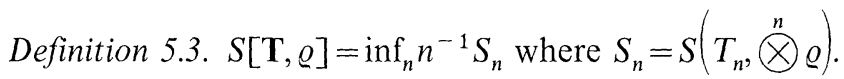

Theorem 5.4. $S[\mathbf{T}, \varrho]=\lim _{n \rightarrow \infty} n^{-1} S_{n} . S[\mathbf{T}, \varrho]$ is an affine function on the convex set of QSPS with a given invariant state $\varrho$.

Proof. By Lemma A.2 and Theorem 5.2 there is an affine map

$$
T_{n} \mapsto \tau_{n}=\tau\left(T_{n}, \bigotimes^{n} \varrho\right) \in S\left(\mathscr{A}_{n} \otimes \mathscr{A}_{n}\right)
$$

such that $S_{n}=S\left(\tau_{n}\right)$. It is easy to check that the density operators $\left\{\tau_{n}\right\}$ form a translation invariant state on the quasilocal algebra of a quantum lattice system over $\mathbb{Z}$. From the result (7.2.11) in [24] it then follows that the limit exists, equals $\inf _{n} n^{-1} S_{n}$, and is an affine function.

Remarks. 1. Obviously $S[\mathbf{T}, \varrho] \leqq S_{1}$. If equality holds then it follows from the triangle inequality [23] that $S_{n}=n S_{1}$ for all $n$ and $\tau_{n}=\bigotimes^{n} \tau_{1}$. This means that $T_{n}=\bigotimes^{n} T_{1}$. Consequently the system is Markovian iff $S[\mathbf{T}, \varrho]=S_{1}$.

2. Our definition of the entropy of a QSP may be compared with the noncommutative entropies introduced by Connes and Størmer [25] and Emch [26]. These authors use a non-commutative counterpart of partitions and thus generalize the Kolmogorov-Sinai entropy to quantum systems without having a construction 
corresponding to our QSP concept. As we have seen our definition coincides with the mean entropy for a quantum lattice system. It is known, however, that the Kolmogorov-Sinai invariant coincides with the mean entropy for classical lattice systems [27]. Therefore it is not unreasonable to consider our entropy definition to be a non-commutative analogy of the Kolmogorov-Sinai entropy.

\section{Appendix}

Let $\mathscr{A}=B(\mathscr{H}), \mathscr{B}=B\left(\mathscr{H}^{\prime}\right)$. A normal CP map $T \in \mathrm{CP}(\mathscr{A}, \mathscr{B})_{\sigma}$ (in the notation of [4]) has a Stinespring representation $(\pi, V)\left(\pi(\mathscr{A}) \subset B(\mathscr{K}), V \in B\left(\mathscr{H}^{\prime}, \mathscr{K}\right), \mathscr{K}\right.$ a Hilbert space)

$$
T(X)=V^{+} \pi(X) V=\sum_{i=1}^{v} V_{i}^{+} X V_{i}
$$

where $V_{i} \in B\left(\mathscr{H}^{\prime}, \mathscr{H}\right)$, i.e. we can choose $\pi$ to be a direct sum $\pi=\bigoplus^{v} \pi_{i}, \pi_{i}(X)=X[28$, 29]. We also find that $\mathscr{K}=\mathscr{H} \otimes h$ where $h$ is a $v$-dimensional Hilbert space and consequently that

$$
B(\mathscr{K})=\mathscr{A} \otimes B(h), \pi(\mathscr{A})=\mathscr{A} \otimes I, \pi(\mathscr{A})^{\prime}=I \otimes B(h)
$$

We will always choose $(\pi, V)$ to be minimal i.e. such that $\left[\pi(\mathscr{A}) V \mathscr{H}^{\prime}\right]=\mathscr{K}[30]$. Then (A.1), with the given $\pi$, is unique up to a unitary transformation [30]

$$
V \mapsto W V, \quad W \in \pi(\mathscr{A})^{\prime}
$$

Now let $\mathscr{A}_{i}=B\left(\mathscr{H}_{i}\right), \mathscr{B}_{i}=B\left(\mathscr{H}_{i}^{\prime}\right), i=1$, 2. Given $T_{i} \in \mathrm{CP}\left(\mathscr{A}_{i}, \mathscr{B}_{i}\right)_{\sigma}$ we can define a unique normal $\mathrm{CP}$ map $T_{1} \otimes T_{2}$ on the $W^{*}$-tensor product $\mathscr{A}_{1} \otimes \mathscr{A}_{2}$ such that

$$
T_{1} \otimes T_{2}\left(X_{1} \otimes X_{2}\right)=T_{1}\left(X_{1}\right) \otimes T_{2}\left(X_{2}\right)
$$

for all $\left\{X_{i} \in \mathscr{A}_{i}\right\}$ through the representation $(\pi, V)$ where

$$
\pi=\pi_{1} \otimes \pi_{2}, \quad V=V_{1} \otimes V_{2}
$$

([31] I.4.5).

Lemma A.1. (a) Let

$$
T:\left(X_{1}, X_{2}\right) \mapsto T\left[X_{1}^{+} ; X_{2}^{+} X_{2} ; X_{1}\right] \in B(\mathscr{H}), \quad\left(X_{i} \in \mathscr{A}_{i}\right)
$$

be a sesquilinear map which is CP in the sense of (2.2). Then there is a Hilbert space $\mathscr{K}_{1}, a$ linear map

$$
V_{1}: \mathscr{A}_{1} \rightarrow B\left(\mathscr{H}, \mathscr{K}_{1}\right) \quad \text { with } \quad \mathscr{K}_{1}=\left[V_{1}(\mathscr{A}) \mathscr{H}\right]
$$

and a CP map $T_{2}: \mathscr{A}_{2} \rightarrow B\left(\mathscr{K}_{1}\right), T_{2}(I)=I$, such that

$$
T\left[X_{1}^{+} ; X_{2} ; X_{1}\right]=V_{1}\left(X_{1}\right)^{+} T_{2}\left(X_{2}\right) V_{1}\left(X_{1}\right) .
$$

If $T$ is ultrastrongly continuous in $X_{2}$ then $T_{2}$ can be chosen to be normal. Note that here $\mathscr{A}_{1}$ can be any linear space with involution.

(b) Let $T \in \mathrm{CP}\left(\mathscr{A}_{1} \otimes \mathscr{A}_{2}, \mathscr{B}_{1} \otimes \mathscr{A}_{2}\right)_{\sigma}$ be such that $T \mid \mathscr{A}_{1} \otimes I_{2}=T_{1} \otimes I_{2}$ where $T_{1} \in \mathrm{CP}\left(\mathscr{A}_{1}, \mathscr{B}_{1}\right)_{\sigma}, I_{i}=$ identity in $\mathscr{A}_{i}$, and let $(\pi, V)$ and $\left(\pi_{1}, V_{1}\right)$ be representations of $T$, 
$T_{1}$ in $\mathscr{K}, \mathscr{K}_{1}$. Then there is an isometry $V_{2} \in \mathscr{A}_{2} \otimes B\left(h_{1}, h\right)$ such that

$$
V=\left(I_{1} \otimes V_{2}\right)\left(V_{1} \otimes I_{2}\right) .
$$

Conversely, given such $T_{1}$ and $V_{2}, T$ has the stated properties.

(c) If, in addition to (b), $\mathscr{A}_{2}=\mathscr{A}_{1} \otimes \mathscr{B}_{2}$ then $T$ defines a normal CP map $T_{2}: \mathscr{A}_{2} \rightarrow B\left(\mathscr{K}_{1}\right) \otimes \mathscr{B}_{2}$ such that $T_{2}(I)=I$ and

$$
\hat{T}\left[X_{1}^{\prime} ; X_{2}^{\prime} X_{2} ; X_{1}\right]=\left(V_{1}^{+} \pi_{1}\left(X_{1}^{\prime}\right) \otimes I_{2}^{\prime}\right) T_{2}\left(X_{2}^{\prime} X_{2}\right)\left(\pi_{1}\left(X_{1}\right) V_{1} \otimes I_{2}^{\prime}\right) \in \mathscr{B}_{1} \otimes \mathscr{B}_{2}
$$

$\left(I_{i}^{\prime}=\right.$ unit in $\mathscr{B}_{i}$ ) defines a map which is $\mathrm{CP}$ in the sense of (2.2). $\hat{T}$ is uniquely determined by $T$ and the map $T \mapsto \hat{T}$ is affine and order-preserving on the convex set of CP maps with the natural partial order.

Proof. (a) Let $T_{1}\left[X^{+}, X\right]=T\left[X^{+} ; I ; X\right]$. The CP property (2.2) means that $T$ and $T_{1}$ are positive definite in the sense of [20] Chapt. 1. By [20] Theorem 1.9 and Lemma 1.4 there are minimal Kolmogorov decompositions

$$
T_{1}\left[X^{+}, X\right]=V_{1}(X)^{+} V_{1}(X), T\left[X_{1}^{+} ; X_{2}^{+} X_{2} ; X_{1}\right]=V\left(X_{1}, X_{2}\right)^{+} V\left(X_{1}, X_{2}\right)
$$

where $V_{1}, V$ are linear maps

$$
V_{1}: \mathscr{A}_{1} \rightarrow B\left(\mathscr{H}, \mathscr{K}_{1}\right), \quad V: \mathscr{A}_{1} \times \mathscr{A}_{2} \rightarrow B\left(\mathscr{H}, \mathscr{K}_{2}\right)
$$

which are unique up to unitary equivalence. By [20] Lemma 1.4 there is a unique isometry $V_{2} \in B\left(\mathscr{K}_{1}, \mathscr{K}_{2}\right)$ such that

$$
V\left(X_{1}, I_{2}\right)=V_{2} V_{1}\left(X_{1}\right) \text {. }
$$

$\pi_{2}$ is uniquely defined by $\pi_{2}\left(X_{2}\right) V\left(X_{1}, I_{2}\right)=V\left(X_{1}, X_{2}\right)$ (cf. [20] Theorem 2.11) and $\left(\pi_{2}, V_{2}\right)$ defines $T_{2}$. The normality property follows as in [29] Theorem 3.3.

(b) In (a) we make the substitutions

$$
\begin{aligned}
& V_{1}\left(X_{1}\right) \mapsto \pi_{1}\left(X_{1}\right) V_{1} \otimes I_{2}, \quad V\left(X_{1}, X_{2}\right) \mapsto \pi\left(X_{1} \otimes X_{2}\right) V \\
& V_{2} \mapsto W \in B\left(\mathscr{K}_{1} \otimes \mathscr{H}_{2}, \mathscr{K}\right)=\mathscr{A}_{1} \otimes \mathscr{A}_{2} \otimes B\left(h_{1}, h\right) .
\end{aligned}
$$

From the minimality property follows that $W\left(\pi_{1}\left(X_{1}\right) \otimes I_{2}\right)=\pi\left(X_{1} \otimes I_{2}\right) W$ and from (A.2) that $W$ is of the form

$$
W=I_{1} \otimes V_{2} \in I_{1} \otimes \mathscr{A}_{2} \otimes B\left(h_{1}, h\right) .
$$

The converse is obvious.

(c) By (b) there is a $T_{2} \in \mathrm{CP}\left(\mathscr{A}_{2}, \mathscr{A}_{2} \otimes B\left(\hbar_{1}\right)\right)_{\sigma}$, which is unique if $\left(\pi_{1}, V_{1}\right)$ is given, defined by

$$
I_{1} \otimes T_{2}\left(X_{2}\right)=W^{+} \pi\left(I_{1} \otimes X_{2}\right) W
$$

and by $\left(\right.$ A.2) $B\left(\mathscr{K}_{1}\right) \otimes \mathscr{B}_{2}=\mathscr{A}_{2} \otimes B\left(h_{1}\right) . \hat{T}$ as defined above is obviously CP. It is also independent of the arbitrariness in $\left(\pi_{1}, V_{1}\right)$ given by (A.3) and is consequently uniquely defined by $T$.

If $T^{\prime} \leqq T$ and $T^{\prime} \mid \mathscr{A}_{1} \otimes I_{2}=T_{1}^{\prime} \otimes I_{2}\left(T_{1}^{\prime} \leqq T_{1}\right)$ then, by an argument similar to (a), (b) but using [20] Theorem 1.12, it follows that $T^{\prime}$ is of the form

$$
T^{\prime}\left(X_{1}^{+} X_{1} \otimes X_{2}\right)=V_{1}\left(X_{1}\right)^{+}\left(I_{1} \otimes T_{2}^{\prime}\left(X_{2}\right)\right) V_{1}\left(X_{1}\right),
$$


where $T_{2}^{\prime} \leqq T_{2}$. Consequently there is an affine order-preserving map defined by the preceding construction

$$
\begin{aligned}
& \left\{T \in \mathrm{CP}\left(\mathscr{A}_{1} \otimes \mathscr{A}_{2}, \mathscr{B}_{1} \otimes \mathscr{A}_{2}\right)_{\sigma}: T \mid \mathscr{A}_{1} \otimes I_{2} \leqq T_{1} \otimes I_{2}\right\} \\
& \rightarrow\left\{T_{2} \in \mathrm{CP}\left(\mathscr{A}_{2}, \mathscr{A}_{2} \otimes B\left(h_{1}\right)\right)_{\sigma}: T_{2}(I) \leqq I\right\}
\end{aligned}
$$

which induces an affine order-preserving map $T \mapsto \hat{T}$.

Lemma A.2. Let $\varrho \in S(\mathscr{H})$ be faithful and $\mathscr{H}^{\prime} \simeq \mathscr{H}$. Then there is a bijective affine map

$$
\left\{T \in \mathrm{CP}(\mathscr{A})_{\sigma}: T(I)=I\right\} \rightarrow\left\{\tau \in S\left(\mathscr{H} \otimes \mathscr{H}^{\prime}\right): \operatorname{Tr}_{\mathscr{H}} \tau=\varrho\right\}
$$

denoted $T \mapsto \tau(T, \varrho)$ which is defined through

$$
\operatorname{Tr}\left[\varrho^{1 / 2} Y^{\sim} \varrho^{1 / 2} T(X)\right]=\tau(T, \varrho)[X \otimes Y],
$$

where $X \in \mathscr{A}, Y \in \mathscr{B}$ and $\sim$ denotes the transposition in any basis in which $\varrho$ is diagonal.

Proof. Using the argument of [32] Lemmas 3.1 and 3.2 we find that for every $\tau \in S\left(\mathscr{H} \otimes \mathscr{H}^{\prime}\right)$

$$
\operatorname{Tr}\left[Y^{\sim} T^{\prime}(X)\right]=\tau[X \otimes Y]
$$

defines a CP map $T^{\prime}$ from $\mathscr{A}$ to the trace class operators on $\mathscr{H}$ such that $T^{\prime}(I)=\varrho$ and vice versa.

$$
T(X)=\varrho^{-1 / 2} T^{\prime}(X) \varrho^{-1 / 2}
$$

defined by a limiting procedure is $\mathrm{CP}$, and it is bounded as $T(I)=I$ i.e. $T \in \mathrm{CP}(\mathscr{A})$. The normality is obvious.

Remark. $\tau(T, \varrho)=\left(T^{*} \otimes I\right)[\tau(\varrho)]$ where $\tau(\varrho)=\tau(I, \varrho)$ is easily seen to be a pure state. $T^{*}(\varrho)=\operatorname{Tr}_{\mathscr{H}}, \tau$, hence $\varrho$ is invariant iff the last quantity is equal to $\varrho$.

Acknowledgements. Most of this work was done at the Department of Mathematics at Bedford College, London, with financial support from the Royal Society. I want to express my thanks to Professor R. F. Streater for the hospitality shown to me there, and to Dr. E. B. Davies for hospitality and interesting discussions during a visit to the Mathematical Institute, Oxford.

\section{References}

1. Davies, E.B.: Quantum theory of open systems. London: Wiley 1976

2. Davies, E.B. : Commun. math. Phys. 39, 91-110 (1974); Math. Ann. 219, 147-158 (1976); Ann. Inst. Henri Poincaré 11, 265-273 (1975)

3. Ingarden, R.S., Kossakowski, A.: Ann. Phys. 89, 451-485 (1975)

4. Lindblad, G.: Commun. math. Phys. 48, 119-130 (1976)

5. Lindblad, G.: Letters Math. Phys. 1, 219-224 (1976)

6. Gorini, V., Kossakowski, A., Sudarshan, E.C.G. : J. Math. Phys. 17, 821-825 (1976)

7. Nakajima, S.: Progr. Theor. Phys. 20, 948-959 (1958)

8. Zwanzig, R.: Lect. Theor. Phys. (Boulder) 3, 106-141 (1960); Physica 30, 1109-1123 (1964)

9. Haake, F.: Springer Tracts Mod. Phys. 66, 98-165 (1973)

10. Davidson, R., Kozak, J.J.: J. Math. Phys. 11, 189-202 (1970)

11. Kubo, R.: J. Phys. Soc. Japan 26, Suppl. 1-5 (1969)

12. Preston, C.: Random fields. In: Lecture notes in mathematics, Vol. 534. Berlin, Heidelberg, New York: Springer 1976 
13. Accardi, L. : Non commutative Mark of chains. Proceedings of the School in Mathematical Physics, Camerino (1974)

14. Accardi, L.: Adv. Math. 20, 329-366 (1976)

15. Haken, H., Weidlich, W.: Z. Physik 205, 96-102 (1967)

16. Lax, M.: Phys. Rev. 172, 350-361 (1968)

17. Haake, F.: Phys. Rev. 3A, 1725-1734 (1971)

18. Lugiato, L.A.: Physica 85A, 18-27 (1976)

19. Bausch, R., Stahl, A.: Z. Physik 204, 32-46 (1967)

20. Evans, D.E., Lewis, J.T.: Comm. Dubl. Inst. Adv. Studies, Ser. A, 24 (1977)

21. Evans, D.E., Lewis, J.T.: Commun. math. Phys. 50, 219-227 (1976)

22. Sakai, S. : $C^{*}$-algebras and $W^{*}$-algebras. Berlin, Heidelberg, New York: Springer 1971

23. Wehrl, A.: Rev. Mod. Phys. 50, 221-260 (1978)

24. Ruelle, D. : Statistical mechanics. New York: Benjamin 1969

25. Connes, A., Størmer, E.: Acta Math. 134, 289-306 (1975)

26. Emch, G.G. : Commun. math. Phys. 49, 191-216 (1976)

27. Robinson, D.W., Ruelle, D.: Commun. math. Phys. 5, 288-300 (1967)

28. Stinespring, W.F.: Proc. Am. Math. Soc. 6, 211-216 (1955)

29. Kraus, K.: Ann. Phys. 64, 311-335 (1970)

30. Arveson, W.: Acta Math. 123, 141-224 (1969)

31. Dixmier, J.: Les algebres d'operateurs dans l'espace Hilbertien. Paris: Gauthier-Villars 1969

32. Lance, C.: J. Funct. Anal. 12, 157-176 (1973)

Communicated by H. Araki

Received March 14, 1978; in revised form November 1. 1978 